\title{
Arsenic exposure in the Canadian general population: levels of arsenic species measured in urine, and associated demographic, lifestyle or dietary factors
}

\author{
A. St-Amand, S. Karthikeyan, M. Guay, R. Charron, A. Vezina \& K. Werry \\ Health Canada
}

\begin{abstract}
In Canada, the primary source of exposure to arsenic is food, followed by drinking water, soil, and air. Measuring inorganic arsenic in urine provides a measure of internal dose integrating all sources and routes of exposure. Urine samples were collected through the Canadian Health Measures Survey (CHMS) in 2009 to 2011 from 2538 respondents and analyzed for various inorganic related arsenic species (arsenite, arsenate, monomethylarsonic acid, dimethylarsinic acid, arsenocholine and arsenobetaine). The geometric mean of urinary dimethylarsinic acid (DMA) in the Canadian population was 3.5 (95\% CI: 3.0-4.0) $\mu \mathrm{g} \mathrm{As} \mathrm{L}^{-1}$. Concentrations were significantly higher in children than in adults. Canadians who eat rice once or more per day have higher urinary concentrations. No association was found with sources of drinking water.
\end{abstract}

\section{INTRODUCTION}

Urinary data for inorganic arsenic provides a measure of internal dose integrating all sources and routes of exposure. This study describes nationallyrepresentative concentrations of inorganic related arsenic species in urine in the Canadian population as measured in the Canadian Health Measure Survey (CHMS) 2009-2011. Potential factors influencing exposure data such as age, sex, smoking status as well as dietary factors such as drinking water source, fish, rice and juice consumption are examined.

\section{METHODS/EXPERIMENTAL}

\subsection{Data collection and laboratory analyses}

The CHMS is designed as a cross-sectional survey conducted over 2-year cycle. Urine samples from 2538 Canadians from 3 to 79 years of age were collected in 18 sites across Canada from August 2009 to November 2011. Urine samples were diluted in ammonium carbonate and analyzed for arsenite (III oxidation state), arsenate (V oxidation state), monomethylarsonic acid (MMA), dimethylarsinic acid (DMA), and arsenocholine and arsenobetaine combined using ultra performance liquid chromatography (UPLC) on a Waters Acquity UPLC coupled to ICP-MS on a Varian 820-MS (INSPQ, 2009).

\subsection{Statistical analysis}

The data were analyzed with SAS 9.2 and SUDAAN 10.0.1 software. All analyses were weighed using the CHMS cycle 2 survey weights (environmental urine subsample) in order to be representative of the Canadian population. Variance estimates were produced using bootstrap weights, taking into account the 13 degrees of freedom. Concentrations that were below LOD were assigned a value of $\mathrm{LOD} / 2$. For each biomarker, descriptive statistics (geometric means and selected percentiles with their associated 95\% confidence intervals) were calculated on the volumetric concentrations.

\section{RESULTS AND DISCUSSION}

\subsection{Descriptive statistics}

Descriptive statistics of arsenic species and metabolites for the Canadian population are presented in Table 1. Inorganic arsenic species and metabolites were detected in $24.4 \%$ of samples as arsenite, $0.5 \%$ as arsenate, $27 \%$ as MMA and $96.2 \%$ as DMA. The geometric mean of DMA in the Canadian population was 3.5 (95\%CI: 3.0-4.0) $\mu \mathrm{g} \mathrm{As} \mathrm{L}^{-1}$.

\subsection{Simple regression model}

Models were developed for the sum of urinary MMA, DMA and arsenite. The results of simple regression models show that concentrations of the sum were significantly associated with rice consumption (higher concentrations in individuals with more than twice per week consumption), fish and shellfish consumption (higher concentrations in fish and shellfish eaters), detection of arsenobetaine and arsenocholine (higher 
Table 1. Geometric means and 95th percentiles of urinary speciated arsenic concentrations in $\mu \mathrm{g} \mathrm{As} \mathrm{L}^{-1}$ (95\% confidence interval) for the Canadian population aged 3-79 years, Canadian Health Measures Survey cycle 2, 2009-2011.

\begin{tabular}{lllll}
\hline Species & Sample size & \% detects & Geometric mean & 95th percentile \\
\hline Arsenite & 2537 & 245 & - & $2.7^{\mathrm{c}}(1.3-4.0)$ \\
Arsenate & 2538 & $<1$ & - & $<$ LOD \\
MMA & 2538 & 27 & - & $1.6(1.2-2.0)$ \\
DMA & 2538 & 96 & $3.5(3.0-4.0)$ & $16^{\mathrm{c}}(7.3-26)$ \\
Arsenocholine, arsenobetaine & 2538 & 51 & - & $48^{\mathrm{c}}(30-67)$ \\
\hline
\end{tabular}

DMA: dimethylarsinic acid; MMA: monomethylarsinic acid.

${ }^{a}$ Limits of detection were: $0.7 \mu \mathrm{g} \mathrm{As} \mathrm{L}^{-1}$ for arsenite, arsenate, MMA and DMA, and 1.5 for arsenocholine/arsenobetaine, respectively.

${ }^{\mathrm{b}}$ If detection was $\leq 60 \%$, the geometric mean was not calculated.

${ }^{\mathrm{c}}$ Data are used with caution as coefficient of variation is between $16.6 \%$ to $33.3 \%$.

concentrations in individuals with detected levels), and ethnic background.

\subsection{Multiple regression model}

Multiple regression models for the sum of MMA and DMA show that LSGM concentrations were significantly higher in 3-5 and 6-11 years old than in 20-79 years old adults $(3-5$ vs $20-79 \mathrm{p}$-value $=0.01,6-11 \mathrm{vs}$ $20-79 \mathrm{p}$-value $<0.0006$ ). The $\mathrm{R}^{2}$ was 0.50 , meaning that $50 \%$ of the variability in the sum of MMA and DMA was explained by the model.

\section{CONCLUSIONS}

This nationally representative dataset reflects background exposure for the Canadian general population which is consistent with levels measured in the US population (Caldwell et al., 2009). Models indicate that sources of exposure to inorganic arsenic in the diet are quite varied but that rice and seafood are among the larger contributors. However, there are others such as vegetables, fruits and fruit juices, other grain products that can contribute to dietary inorganic arsenic exposure. Approximately a third of dietary inorganic arsenic exposure is methylated arsenic. A high proportion DMA in urine could be from direct ingestion and not inorganic arsenic metabolism.

\section{ACKNOWLEDGEMENTS}

This study was funded under the Government of Canada's Chemicals Management Plan. We would like to thank Statistics Canada for planning and Centre de Toxicologie du Québec for conducting the laboratory analyses.

\section{REFERENCES}

Caldwell, K.L., Jones, R.L., Verdon, C.P., Jarrett, J.M., Caudill, S.P. \& Osterloh, J.D. 2009. Levels of urinary total and speciated arsenic in the US population: National Health and Nutrition Examination Survey 2003-2004. J. Expo. Sci. Env. Epid. 19(1): 59-68.

INSPQ 2009. Analytical method for the determination of arsenic species in urine by ultra-performance liquid chromatography coupled to argon plasma induced mass spectrometry (HPLC-ICP-MS) (M-585), condensed version for CHMS. Laboratoire de Toxicologie, Québec, QC. 